PROCEEDINGS OF THE

AMERICAN MATHEMATICAL SOCIETY

Volume 33, Number 2, June 1972

\title{
ANNIHILATOR IDEALS IN THE COHOMOLOGY OF BANACH ALGEBRAS
}

\author{
A. M. SINCLAIR
}

Abstract. If $A$ is a $C^{*}$-algebra, if $X$ is a Banach $A$-module, and if $I$ is the annihilator of $X$ in $A$, then the cohomology space $\mathscr{H}^{n}\left(A, X^{*}\right)$ is isomorphic to $\mathscr{H}^{n}\left(A / J, X^{*}\right)$ for each positive integer $n$.

B. E. Johnson [5, Proposition 1.8] has shown that if $A$ is a Banach algebra with a bounded approximate identity and if $X$ is a Banach $A$ module, then $X_{1}=\{a x b: x \in X ; a, b \in A\}$ is a closed neo-unital submodule of $X$ and $\mathscr{H}^{n}\left(A, X^{*}\right)$ is isomorphic to $\mathscr{H}^{n}\left(A, X_{1}^{*}\right)$. In particular this result shows that in calculating cohomologies of dual Banach modules for $C^{*}$-algebras attention may be restricted to neo-unital modules. Since each closed (two-sided) ideal in a $C^{*}$-algebra has a bounded approximate identity [2, Propositions 1.8.2 and 1.7.2] our result shows that for $C^{*}$ algebras and dual Banach modules attention may be restricted to faithful modules. If $A$ is a Banach algebra, if $X$ is a Banach $A$-module, and if $J$ is a closed ideal in $A$ annihilating $X$, then there is a natural homomorphism $Q$, which is defined in Theorem 1 , from $\mathscr{H}^{n}\left(A \mid J, X^{*}\right)$ into $\mathscr{H}^{n}\left(A, X^{*}\right)$. Under the additional assumption that $J$ has a bounded approximate identity, this homomorphism $Q$ is an isomorphism (Theorem 1). In Remark 4 we give an elementary example to show that an additional assumption on $J$ is necessary if the conclusion of Theorem 1 is to hold.

I am grateful to B. E. Johnson for a preprint of [5], and I acknowledge a C.S.I.R. travel grant.

If $A$ is a Banach algebra, if $X$ is a Banach $A$-module, and if $n$ is a positive integer, we let $\mathscr{L}^{n}\left(A, X^{*}\right)$ denote the Banach space of continuous $n$-linear mappings from $A$ into $X^{*}$, the dual of $X$ (our notation and definitions are from [5]). Recall that $\mathscr{L}^{n}\left(A, X^{*}\right)$ is the dual space of a Banach space $A \hat{\hat{\otimes}} A \hat{\hat{\otimes}} \cdot \cdots \hat{\otimes} A \hat{\otimes} X$ (see [5]). We also give $X^{*}$ the dual $A$-module structure from the Banach $A$-module $X$ by defining af and fa for $a$ in $A$ and $f$ in $X^{*}$ by

$$
\langle a f, x\rangle=\langle f, x a\rangle \text { and }\langle f a, x\rangle=\langle f, a x\rangle
$$

Received by the editors August 9, 1971.

AMS 1970 subject classifications. Primary 46H25, $18 \mathrm{H} 15$.

Key words and phrases. Banach algebra, Banach module, cohomology, annihilator ideal, approximate identity, $C^{*}$-algebra.

(c) American Mathematical Society 1972 
for all $x$ in $X$. The mapping $\delta^{n}$ from $\mathscr{L}^{n-1}\left(A, X^{*}\right)$ to $\mathscr{L}^{n}\left(A, X^{*}\right)$ is defined by

$$
\begin{aligned}
\left(\delta^{n} T\right)\left(a_{1}, \cdots, a_{n}\right)= & a_{1} T\left(a_{2}, \cdots, a_{n}\right) \\
& +\sum_{j=1}^{n-1}(-1)^{j} T\left(a_{1}, \cdots, a_{j} a_{j+1}, \cdots, a_{n}\right) \\
& +(-1)^{n} T\left(a_{1}, \cdots, a_{n-1}\right) a_{n}
\end{aligned}
$$

for $T$ in $\mathscr{L}^{n-1}\left(A, X^{*}\right)$, which we take to be $X^{*}$ when $n-1=0$, and for $a_{1}, \cdots, a_{n}$ in $A$. Then $\delta^{n+1} \delta^{n}=0$, and we let

$$
\mathscr{H}^{n}\left(A, X^{*}\right)=\operatorname{Ker} \delta^{n+1} / \operatorname{Im} \delta^{n} .
$$

We use the same $\delta^{n}$ for all algebras and modules. We say that an ideal $J$ in a Banach algebra $A$ annihilates a Banach $A$-module $X$ if $a x=x a=0$ for all $a$ in $J$ and $x$ in $X$. If the closed ideal $J$ annihilates $X$, we regard $X$ as a Banach $A / J$-module by defining $(a+J) x=a x$ and $x(a+J)=x a$ for all $a$ in $A$ and $x$ in $X$.

TheORem 1. Let $A$ be a Banach algebra, let $X$ be a Banach A-module, and let $J$ be a closed ideal in $A$ annihilating $X$. If $J$ has a bounded approximate identity, then $\mathscr{H}^{n}\left(A / J, X^{*}\right)$ is isomorphic to $\mathscr{H}^{n}\left(A, X^{*}\right)$ under the mapping

$$
Q: T+\operatorname{Im} \delta^{n} \rightarrow \theta T+\operatorname{Im} \delta^{n}
$$

where $(\theta T)\left(a_{1}, \cdots, a_{n}\right)=T\left(a_{1}+J, \cdots, a_{n}+J\right)$ for $T$ in $\mathscr{L}^{n}\left(A / J, X^{*}\right)$ and $a_{1}, \cdots, a_{n}$ in $A$.

We require a lemma before proving Theorem 1 . Under the hypotheses of Theorem 1 we shall regard $\mathscr{L}^{1}\left(A \mid J, X^{*}\right)$ as a Banach $A$-module by defining $a T$ and $T a$, for all $a$ in $A$ and $T$ in $\mathscr{L}^{1}\left(A / J, X^{*}\right)$, by

$$
\begin{aligned}
& (a T)(b+J)=a T(b+J) \text { and } \\
& (T a)(b+J)=T(a b+J)-T(a+J) b
\end{aligned}
$$

for all $b$ in $A$. Compare the following lemma with $[5,1 . a]$.

Lemma 2. Let $A$ be a Banach algebra, let $X$ be a Banach A-module, and let $J$ be a closed ideal in $A$ annihilating $X$. Let $n$ be an integer greater than 1. If $J$ has a bounded approximate identity, then $\mathscr{H}^{n-1}\left(A, \mathscr{L}^{1}\left(A / J, X^{*}\right)\right)$ is isomorphic to $\mathscr{H}^{n}\left(A, X^{*}\right)$ under the mapping $\chi: T+\operatorname{Im} \delta^{n-1} \rightarrow \psi_{n} T+\operatorname{Im} \delta^{n}$ where

$$
\left(\psi_{n} T\right)\left(a_{1}, \cdots, a_{n}\right)=T\left(a_{1}, \cdots, a_{n-1}\right)\left(a_{n}+J\right)
$$

for all $T$ in $\mathscr{L}^{n-1}\left(A, \mathscr{L}^{1}\left(A \mid J, X^{*}\right)\right)$ and all $a_{1}, \cdots, a_{n}$ in $A$. 
Proof. A routine calculation using equations (1), (2), and (3) shows that

$$
\psi_{n+1} \delta^{n}=\delta^{n+1} \psi_{n}
$$

for $n$ a positive integer. Applying equation (4) with $n$ replaced by $n-1$ we observe that the mapping $\chi$ is well defined. Using (4) as it stands we observe that $\chi$ maps $\mathscr{H}^{n-1}\left(A, \mathscr{L}^{1}\left(A / J, X^{*}\right)\right)$ into $\mathscr{H}^{n}\left(A, X^{*}\right)$. We now use the bounded approximate identity in $J$ to show that $\chi$ is an isomorphism.

We shall show that there is an $R$ in $\mathscr{L}^{n-1}\left(A, X^{*}\right)$ with

$$
\left(T-\delta^{n} R\right)\left(a_{1}, \cdots, a_{n}\right)=0
$$

if $a_{n}$ is in $J$. If we have found such an $R$, then equation (5) implies that $T-\delta^{n} R$ is in the image of $\psi_{n}$, and so there is a $P$ in $\mathscr{L}^{n-1}\left(A, \mathscr{L}^{1}\left(A / J, X^{*}\right)\right)$ with $\psi_{n} P=T-\delta^{n} R$. From equation (4) we obtain

$$
\psi_{n+1} \delta^{n} P=\delta^{n+1} \psi_{n} P=\delta^{n+1}\left(T-\delta^{n} R\right)=0,
$$

and thus $\delta^{n} P=0$ because $\psi_{n+1}$ is a monomorphism. Therefore $\chi$ is an epimorphism.

Let $T$ be in $\mathscr{L}^{n}\left(A, X^{*}\right)$ with $\delta^{n+1} T=0$, and let $\left\{e_{\alpha}\right\}$ be a bounded approximate identity in $J$. Now $\mathscr{L}^{n}\left(A, X^{*}\right)$ may be regarded as the dual space of $A \hat{\otimes} \cdots \hat{\otimes} A \hat{\otimes} X$, where there are $n$-copies of $A$, and under this identification the weak-*-topology on $\mathscr{L}^{n}\left(A, X^{*}\right)$ is generated by the seminorms $S \rightarrow\left\langle S\left(a_{1}, \cdots, a_{n}\right), x\right\rangle$ where $\langle\cdot, \cdot\rangle$ denotes the pairing of $X$ and $X^{*}$, and $a_{1}, \cdots, a_{n}$ are in $A$ and $x$ is in $X$ (see [5, §1]). Now $\left\{T\left(\cdot, \cdots, e_{\alpha}\right)\right\}$ is a bounded net in $\mathscr{L}^{n-1}\left(A, X^{*}\right)$ and hence has a subnet convergent in the weak-*-topology of $\mathscr{L}^{n-1}\left(A, X^{*}\right)$. For convenience, we take $\left\{T\left(\cdot, \cdots, e_{\alpha}\right)\right\}$ itself to be convergent in the weak-*-topology to an element $(-1)^{n+1} R$ in $\mathscr{L}^{n-1}\left(A, X^{*}\right)$. All limits in this proof are over the directed set corresponding to the net $\left\{e_{\alpha}\right\}$ and are in the weak-*-topology in $X^{*}$.

Using $\delta^{n+1} T\left(a_{1}, \cdots, a_{n}, e_{\alpha}\right)=0$, equation (1), and the definition of $R$, we obtain

$$
\begin{aligned}
& \delta^{n} R\left(a_{1}, \cdots, a_{n}\right) \\
& =(-1)^{n+1} \lim \left\{a_{1} T\left(a_{2}, \cdots, a_{n}, e_{\alpha}\right)+\sum_{j=1}^{n-1}(-1)^{j} T\left(a_{1}, \cdots, a_{j} a_{j+1}, \cdots, a_{n}, e_{\alpha}\right)\right. \\
& \left.\quad+(-1)^{n} T\left(a_{1}, \cdots, a_{n-1}, e_{\alpha}\right) a_{n}\right\} \\
& =(-1)^{n} \lim \left\{(-1)^{n} T\left(a_{1}, \cdots, a_{n-1}, a_{n} e_{\alpha}\right)+(-1)^{n+1} T\left(a_{1}, \cdots, a_{n}\right) e_{\alpha}\right. \\
& \left.+(-1)^{n+1} T\left(a_{1}, \cdots, a_{n-1}, e_{\alpha}\right) a_{n}\right\} \\
& =T\left(a_{1}, \cdots, a_{n}\right)
\end{aligned}
$$

provided $a_{n}$ is in $J$. This proves equation (5). 
We shall now prove that $\chi$ is one-to-one. We let $T$ be in

$$
\mathscr{L}^{n-1}\left(A, \mathscr{L}^{1}\left(A / J, X^{*}\right)\right)
$$

with $\psi_{n} T=\delta^{n} S$ where $S$ is some element of $\mathscr{L}^{n-1}\left(A, X^{*}\right)$. We shall obtain an $R$ in $\mathscr{L}^{n-2}\left(A, X^{*}\right)$ such that

$$
\left(S-\delta^{n-1} R\right)\left(a_{1}, \cdots, a_{n-1}\right)=0
$$

if $a_{n-1}$ is in $J$. Having found such an $R$ there is a $P$ in $\mathscr{L}^{n-2}\left(A, \mathscr{L}^{1}\left(A \mid J, X^{*}\right)\right)$ such that $\psi_{n-1} P=S-\delta^{n-1} R$. From this and equation (4) we obtain $\psi_{n} T=\delta^{n} S=\delta^{n} \psi_{n-1} P+\delta^{n} \delta^{n-1} R=\psi_{n} \delta^{n-1} P$. Because $\psi_{n}$ is a monomorphism, $T$ is equal to $\delta^{n-1} P$.

We let $T$ be in $\mathscr{L}^{n-1}\left(A, \mathscr{L}^{1}\left(A / J, X^{*}\right)\right)$ with $\psi_{n} T=\delta^{n} S$ where $S$ is some element of $\mathscr{L}^{n-1}\left(A, X^{*}\right)$. As in the above proof that $\chi$ is an epimorphism, there is a bounded approximate identity $\left\{e_{x}\right\}$ in $J$ and an $R$ in $\mathscr{L}^{n-2}\left(A, X^{*}\right)$ such that

$$
R\left(a_{1}, \cdots, a_{n-2}\right)=(-1)^{n} \lim S\left(a_{1}, \cdots, a_{n-2}, e_{\alpha}\right)
$$

for all $a_{1}, \cdots, a_{n-2}$ in $A$. If $a_{n-1}$ is in $J$ and if $a_{1}, \cdots, a_{n-2}$ are in $A$, then

$$
\begin{aligned}
& \delta^{n-1} R\left(a_{1}, \cdots, a_{n-1}\right) \\
& =(-1)^{n} \lim \left\{a_{1} S\left(a_{2}, \cdots, a_{n-1}, e_{x}\right)+\sum_{j=1}^{n-2}(-1)^{j} S\left(a_{1}, \cdots, a_{j} a_{j+1}, \cdots, a_{n-1}, e_{\alpha}\right)\right. \\
& \left.\quad+(-1)^{n-1} S\left(a_{1}, \cdots, a_{n-2}, e_{\alpha}\right) a_{n-1}\right\} \\
& =(-1)^{n} \lim \left\{\delta^{n} S\left(a_{1}, \cdots, a_{n-1}, e_{x}\right)+(-1)^{n} S\left(a_{1}, \cdots, a_{n-2}, a_{n-1} e_{\alpha}\right)\right\}
\end{aligned}
$$

because $a_{n-1}$ and $e_{\alpha}$ are in $J$, which annihilates $X^{*}$. Since $\delta^{n} S=\psi_{n} T$, it follows that $\delta^{n} S\left(a_{1}, \cdots, a_{n-1}, e_{x}\right)=0$ because $e_{x}$ is in $J$. This completes the proof of the lemma.

Proof OF THEOREM 1. The definitions of $\theta$ and $\delta^{n}$ imply that $\delta^{n} \theta=\theta \delta^{n}$. Thus the mapping $Q$, defined in the statement of the theorem, is a well defined homomorphism from $\mathscr{H}^{n}\left(A / J, X^{*}\right)$ into $\mathscr{H}^{n}\left(A, X^{*}\right)$. We shall prove that $Q$ is an isomorphism by induction on $n$ over all Banach $A$-modules that are annihilated by $J$.

Now we consider $n=1$. If $f$ is in $X^{*}$, then $\delta^{1}(f)(a)=a f-f a=(a+J) f-$ $f(a+J)=\delta^{1}(f)(a+J)=\left(\theta \delta^{1}(f)\right)(a)$ by definition of $\delta^{1}$, so that $\theta \operatorname{Im} \delta^{1}=$ $\operatorname{Im} \delta^{1}$. If $D$ is in $\operatorname{Ker} \delta^{2}$, which is contained in $\mathscr{L}^{1}\left(A, X^{*}\right)$, then $D(a b)=$ $D(a) b+a D(b)$ for all $a, b$ in $A$ by definition of $\delta^{2}$. If $c$ is in $J$, then by Cohen's Factorization Theorem [1] we have $c=a b$ for some $a$ and $b$ in $J$. Thus $D(c)=a D(b)+D(a) b=0$ because $J$ annihilates $X^{*}$. We may now define an operator $T$ in $\mathscr{L}^{1}\left(A / J, X^{*}\right)$ by $T(a+J)=D(a)$ for all $a$ in $A$. Then $\theta=D$, and $\delta^{2} T=0$. This shows that $Q$ is an isomorphism for $n=1$. 
Suppose the result has been proved for $n$. We firstly observe that $\mathscr{L}^{1}\left(A \mid J, X^{*}\right)$ is, as a Banach $A$-module, the dual of the Banach $A$-module $Y=(A \mid J) \hat{S}$, the projective tensor product of Banach spaces [5, $\S 1]$, where we define the module operations on generating elements $(a+J) \hat{\otimes} x$ of the tensor product by

$$
\begin{aligned}
& b((a+J) \hat{\hat{\Theta}} x)=(b a+J) \hat{\otimes} x-(b+J) \hat{\otimes} a x \text { and } \\
& ((a+J) \hat{\hat{\otimes}} x) b=(a+J) \hat{\hat{\vartheta}} x b
\end{aligned}
$$

and lift the definitions to $Y$ by linearity and continuity. Because $J$ annihilates $X$, equations (7) imply that $J$ annihilates the $A$-module $Y$. Now by Lemma 2 , our inductive hypothesis on $n$, and the reduction of dimension lemma for cohomology [5, 1(a)], the following isomorphisms hold:

$$
\begin{aligned}
\mathscr{H}^{n+1}\left(A, X^{*}\right) & \cong \mathscr{H}^{n}\left(A, \mathscr{L}^{1}\left(A \mid J, X^{*}\right)\right) \cong \mathscr{H}^{n}\left(A \mid J, \mathscr{L}^{1}\left(A \mid J, X^{*}\right)\right) \\
& \cong \mathscr{H}^{n+1}\left(A \mid J, X^{*}\right) .
\end{aligned}
$$

Each of these isomorphisms is the natural one arising from the quotient $A / J$. Thus $Q$ is an isomorphism for $n+1$. This completes the proof.

Our corollary generalizes [4, Theorems 4.1 and 4.2] from $n=1$ and 2 to any positive integer $n$.

COROLlaRy 3. Let A be a Banach algebra in which each closed cofinite ideal has a bounded approximate identity. If $X$ is a finite dimensional Banach $A$-module and $n$ is a positive integer, then $\mathscr{H}^{n}(A, X)=\{0\}$.

Proof. The annihilator $J$ of $X$ is a closed cofinite ideal in $A$, and $X$ is the dual of the Banach $A$-module $X^{*}$. By Theorem 1, we have $\mathscr{H}^{n}(A, X)$ isomorphic to $\mathscr{H}^{n}(A / J, X)$. An ideal in $A / J$ is of the form $I / J$, where $I$ is a closed cofinite ideal in $A$ containing $J$. Since $I$ has a bounded approximate identity, $I^{2}$ is equal to $I$ by Cohen's Factorization Theorem [1]. This shows that $A / J$ is a finite dimensional semisimple algebra. As every $n$-linear operator from $A / J$ into $X$ is continuous, $\mathscr{H}^{n}(A / J, X)$ coincides with Hochschild's cohomology groups [3] for the $A / J$-module $X$. Hochschild's $n$ th-cohomology group for the $A \mid J$-module $X$ is null [3, Theorem 4.1], and so $\mathscr{H}^{n}(A, X)=\{0\}$.

REMARK 4. We now outline an example which shows that some assumption on $J$ like that of a bounded approximate identity is necessary if the conclusion of Theorem 1 is to hold. Let $X$ be a (finite dimensional) Banach space, and let $X$ have the zero product ( $x y=0$ for all $x, y$ in $X$ ). Let $A$ be the Banach algebra obtained by adjoining an identity to $X$, and let the ideal $J$ be $X$. We regard $X$ as an $A$-module with the natural module operations. Then $A / J$ is equal to $C 1$, and so $\mathscr{H}^{1}\left(A / J, X^{*}\right)$ is zero as may be proved in a number of ways (for example [3, Theorem 4.1]). However 
for the algebra $A$ we obtain $\operatorname{Im} \delta^{1}$ is $\{0\}$, and $\operatorname{Ker} \delta^{2}$ is $\mathscr{L}^{1}\left(J, X^{*}\right)$, so that $\mathscr{H}^{1}\left(A, X^{*}\right)=\mathscr{L}^{1}\left(J, X^{*}\right)$ and the conclusion of Theorem 1 does not hold.

\section{REFERENCES}

1. P. J. Cohen, Factorization in group algebras, Duke Math. J. 26 (1959), 199-205. MR 21 \#3729.

2. J. Dixmier, Les $C^{*}$-algèbres et leurs représentations, Cahiers Scientifiques, fasc. 29, Gauthier-Villars, Paris, 1964. MR 30 \#1404.

3. G. Hochschild, On the cohomology groups of an associative algebra, Ann. of Math. (2) 46 (1945), 58-67. MR 6, 114.

4. B. E. Johnson, The Wedderburn decomposition of Banach algebras with finite dimensional radical, Amer. J. Math. 90 (1968), 866-876. MR 38 \#1529.

5. - Cohomology in Banach algebras, Mem. Amer. Math. Soc. (to appear).

Department of Mathematics, University of Edinburgh, Edinburgh, Scotland

DePARTMENT OF MATHEMATICS, UNIVERSITY OF THE WITWATERSRAND, JohANNESBURG, SOUTH AfRICA (Current address) 